\title{
Erektile Dysfunktion: Stoßwellentherapie und ein neuer PDE-5-Hemmer
}

\begin{abstract}
etztes Jahr auf dem EAU-Kongress in
Wien haben israelische Forscher eine Studie vorgestellt, bei der Patienten mit erektiler Dysfunktion (ED) von einer Behandlung mit Stoßwellen profitierten. Die damalige Patientenpopulation hatte nicht auf Inhibitoren der Phosphodiesterase 5 (PDE-5) angesprochen. Dr. Ilan Gruenwald, Haifa, präsentierte nun Daten von 60 Patienten, bei denen PDE-5-Hemmer vormals gewirkt haben. Die Patienten erhielten in zwölf Sitzungen entweder eine Stoßwellen- oder Scheinbehandlung. Unmittelbar vor der ersten und einen Monat nach der letzten Sitzung wurden verschiedene ED-Parameter bestimmt. Bei 56\% der Patienten aus der aktiven Gruppe bes-
\end{abstract}

serten sich IIEF-Score und flussassoziierte Dilatation (FMD) signifikant, im Vergleich zu 5\% in der Placebo-Gruppe. Gruenwald betonte, dass die Stoßwellen im Unterschied zum Einsatz bei Induratio Penis Plastica mit niedriger Intensität appliziert werden und vollkommen schmerzfrei sind. Er gestand ein, dass der genaue Mechanismus bislang noch nicht geklärt ist, der klinische Effekt der Methode sei jedoch erwiesen.

Für die On-Demand-Therapie der ED könnte mit Avanafil bald ein vierter PDE5-Inhibitor zur Verfügung stehen. Dr. John P. Mulhall, New York, stellte vier verschiedene Phase-II- und -III-Studien zu Avanafil vor. In allen Studien bekamen die Patienten nach einer vierwöchigen Placebo-Auswaschphase randomisiert entweder Avanafil oder Placebo. Die Wirkung setzte teilweise bereits nach $15 \mathrm{Mi}$ nuten ein und hielt bis zu sechs Stunden an. Mulhall erklärte, dass sich Avanafil aufgrund des schnellen Wirkeintritts besonders für die On-Demand-Therapie eigne und dass auch „schwere Fälle“ vom PDE-5-Hemmer profitieren. Zur Studienpopulation zählten unter anderem Diabetiker, prostatektomierte Patienten und Männer, die seit über fünf Jahren an ED litten.

Sebastian Lux

P597, P701, P1023

\section{Überaktive Blase: neue Substanzklasse in der Entwicklung}

D erzeitiger Standard in der Therapie der überaktiven Blase $(\mathrm{OAB})$ sind Anticholinergika. Da Nebenwirkungen wie Mundtrockenheit, Verstopfung und verschwommene Sicht die Compliance einschränken können, wird nach Alternativen gesucht. Gerade für Patienten, für die Mundtrockenheit unerträglich ist, könnte die in der Entwicklung befindliche Substanzklasse der Beta-3-AdrenozeptorAgonisten geeignet sein.

Ein Vertreter, Mirabegron, hat in mehreren Studien seine Wirkung und Verträglichkeit erwiesen und ist in Japan bereits zugelassen. Prof. Chris Chapple, Sheffield, stellte nun Langzeitdaten vor. Sowohl die 50-mg-als auch die 100-mg-Dosis von Mirabegron erzielten bei täglicher Ein- nahme über zwölf Monate ähnlich gute Ergebnisse in puncto Inkontinenzepisoden, Anzahl der täglichen Miktionen und durchschnittlichem Harnvolumen wie die etablierte anticholinerge Therapie. Dr. Vikram Khullar, London, betonte, dass Mirabegron wirksam ist, unabhängig davon, ob die Patienten zuvor mit Anticholinergika behandelt wurden oder nicht. In beiden britischen Studien war die Mundtrockenheit unter Mirabegron auf Placeboniveau, unter Antimuskarinika war sie etwa drei Mal so hoch. Mirabegron erhöht zudem nicht den Augeninnendruck, womit das Glaukom-Risiko gering sein dürfte.

Zur selben Substanzklasse gehört Solabegron. Dr. Eliot Ohlstein, Malvern
(USA), stellte eine randomisierte, doppelblinde, Placebo-kontrollierte Phase-IIStudie mit 224 Patientinnen vor, die unter Dranginkontinenz litten. Nach acht Wochen nahmen die Anzahl der Inkontinenz- und Drangepisoden ab und das durchschnittliche Harnvolumen pro Miktion $\mathrm{zu}$ - beides sowohl gegenüber Baseline als auch Placebo. Die 125-mg-Dosis stellte sich dabei als wirksamer als die 50-mg-Dosis heraus. Häufigste Nebenwirkung waren Kopfschmerzen, die in Verum- und Placebogruppe gleich häufig auftraten (8\%).

Sebastian Lux

P683, P684, P685, P686

\section{Hier muss der Dummy durch a}

